\title{
Acumulación y descomposición de hojarasca en bosques secundarios del sur de la Sierra Madre de Chiapas, México
}

\author{
Litter accumulation and decomposition in secondary forests of the southern \\ Sierra Madre of Chiapas, Mexico
}

\author{
Elsa Saraí Gaspar-Santos a , Mario González-Espinosa a*, \\ Neptalí Ramírez-Marcial a , José David Álvarez-Solís ${ }^{\text {b }}$ \\ *Autor de correspondencia: a El Colegio de la Frontera Sur (ECOSUR), Departamento de Conservación de la Biodiversidad, \\ Carretera Panamericana y Periférico Sur s/n, C.P. 29290, San Cristóbal de Las Casas, Chiapas, México, \\ tel.: +52 967674 9000, ext. 1318, fax: +52967674 9021, mgonzale@ecosur.mx \\ ${ }^{\mathrm{b}}$ El Colegio de la Frontera Sur (ECOSUR), Departamento de Agricultura, Sociedad y Ambiente, \\ San Cristóbal de Las Casas, Chiapas, México.
}

\begin{abstract}
SUMMARY
Litter accumulation and decomposition processes affect organic matter input and cycling of nutrients in forest ecosystems. Forest restoration may enhance these two processes reducing soil erosion and the risks of landslides on steep slopes. The main objective of this study was to identify factors determining litter accumulation and decomposition in degraded secondary forests from the upper part of the Grijalva River watershed (Chiapas, Mexico). We assessed litter accumulation over a period of 290 days on tilted (52-104 \% slope) and horizontal collecting surfaces, and litter decomposition of Alnus acuminata, Quercus segoviensis and Pinus tecunumanii ("litter bag" method) in six localities ( $\geq 5$ replicate $1,000 \mathrm{~m}^{2}$ plots). We used analysis of variance to compare vegetation and soil attributes among forest fragments, and we fitted multiple linear regression models to identify explanatory variables of litter accumulation and decomposition. Litter accumulation on both types of collecting surfaces was higher in SW-faced slopes, and with lower tree density and soil fertility. Litter decomposition of $A$. acuminata (40-56\%) was faster than in the other two species, and was higher in more humid sites at higher elevations, with higher canopy cover and in mountainsides with SW slope-aspect. We propose planting juveniles of A. acuminata (followed by $Q$. segoviensis) in the initial stages of a forest restoration program aimed at improving the structure and stability of soils.
\end{abstract}

Key words: Alnus, Quercus, Pinus, Grijalva River, landslides.

\section{RESUMEN}

La acumulación y descomposición de la hojarasca determinan el aporte de materia orgánica al suelo e influyen en la circulación de nutrimentos. Mediante la restauración forestal, ambos procesos pueden reducir la erosión del suelo y el riesgo de deslizamiento de laderas. El objetivo principal fue identificar determinantes de la acumulación y descomposición de la hojarasca en bosques secundarios degradados de la cuenca alta del río Grijalva (Chiapas, México), y sugerir especies que por la rápida descomposición de su hojarasca sean útiles en la restauración. Se cuantificó la hojarasca acumulada durante 290 días sobre superficies horizontales e inclinadas (52-104 \%) y se evalúo la descomposición de hojarasca de Alnus acuminata, Quercus segoviensis y Pinus tecunumanii (método litter bag) en seis fragmentos forestales ( $\geq 5$ parcelas de $1.000 \mathrm{~m}^{2}$ como repeticiones). Mediante análisis de varianza se compararon atributos topográficos, estructurales y edáficos de los fragmentos, y se ajustaron modelos de regresión lineal múltiple para evaluar determinantes de la acumulación y descomposición de la hojarasca. Sobre ambos tipos de superficie de recolección, la acumulación fue mayor en laderas expuestas al SO y en fragmentos menos densos y con suelos menos fértiles. La descomposición de hojarasca de $A$. acuminata (40-56 \%) fue más rápida que en las otras especies, y fue mayor en sitios más húmedos a mayores elevaciones, con mayor cobertura y en laderas con orientación SO. Se propone plantar A. acuminata (seguida de $Q$. segoviensis) al inicio la restauración con fines de mejorar la estructura y estabilidad del suelo.

Palabras clave: Alnus, Quercus, Pinus, río Grijalva, deslizamiento de laderas.

\section{INTRODUCCIÓN}

Los paisajes forestales proporcionan múltiples servicios ecosistémicos, tales como captación de agua, conservación de la biodiversidad, fertilidad del suelo y regulación climática (Lara et al. 2009). Sin embargo, el efecto de la cobertura del dosel sobre la reducción de las consecuencias de eventos climáticos extremos es materia de debate (Laurance 2007). Por una parte, se argumenta que los deslaves e inundaciones derivados de lluvias extremas no se relacionan con la cobertura arbórea en las partes altas de las cuencas (CIFOR 2005). Por el contrario, se ha documenta- 
do empíricamente que la reducción de la cobertura boscosa se correlaciona con el riesgo y severidad de las inundaciones o deslaves derivados de lluvias intensas (Bradshaw et al. 2007). La función de retención de humedad en el suelo, no sólo depende de la presencia de cobertura arbórea, sino de los atributos físicos y químicos que se desarrollan en el suelo a través de décadas, entre los que destaca la cantidad de humus (Berg y McClaugherty 2008).

El aumento de humus contribuye a mejorar la estructura edáfica e incrementa la capacidad de infiltración, almacenamiento de agua y la conductividad hidráulica en el suelo (Bens et al. 2007). La acumulación y descomposición de la hojarasca y su conversión en humus dependen de factores como la productividad primaria, la composición y la estructura de la vegetación (Kumar y Deepu 1992, Scherer-Lorenzen et al. 2007), los flujos de viento y agua, la temperatura y la humedad en el ambiente, la fertilidad del suelo (Vitousek y Sanford 1986), la actividad de los microorganismos y la fauna edáfica (Lavelle et al. 1993), así como de la inclinación del terreno y su exposición respecto al tránsito del sol (Sariyildiz y Küçük 2008). La relación entre la hojarasca y el humus es de interés particular en los bosques tropicales de montaña debido a que sus ciclos biogeoquímicos son relativamente lentos, además de que sus suelos tienen fertilidad media o baja (Hamilton 1995).

En las regiones montañosas del sur de México, la fragmentación y degradación de diversos tipos de bosques se han combinado con eventos meteorológicos extremos (Richter 2000, Restrepo et al. 2009). Los ciclones Mitch (en 1998) y Stan (en 2005) provocaron en la parte alta de la cuenca del río Grijalva deslizamientos de laderas deforestadas y cambios en el curso de algunos ríos; además, la severa deforestación en esas regiones altas se asoció en esos mismos años, así como en 2007 y 2011, a inundaciones prolongadas en la planicie costera del Golfo de México. Al igual que en otras regiones montañosas, la restauración de las áreas arboladas es considerada de manera prioritaria para reducir los riesgos y la vulnerabilidad de las poblaciones montanas ante los efectos del cambio climático (Chazdon 2008, Lara et al. 2009). A diferencia del establecimiento de plantaciones con fines productivos, en la restauración de bosques se utilizan especies leñosas nativas que puedan reducir la erosión del suelo mediante el aporte y descomposición de su hojarasca y así reducir el impacto de las gotas de lluvia sobre el suelo, reducir la escorrentía y, al mismo tiempo, incrementar la infiltración (Islam et al. 2001).

La predicción de los efectos favorables de la restauración requiere de un conocimiento detallado de las interacciones de factores climáticos, edáficos y de la vegetación, $\mathrm{y}$ a menudo, sus efectos requieren de algunos años para ser evidentes. El objetivo principal de este estudio es evaluar la acumulación y descomposición de la hojarasca en bosques de montaña degradados en función de factores topográficos, estructurales y edáficos en fragmentos fores- tales de seis comunidades de la cuenca alta del río Grijalva (Chiapas, México). Como objetivos particulares se plantean: (1) caracterizar la estructura y composición florística de fragmentos forestales degradados, las propiedades físicas y químicas de los suelos y sus condiciones topográficas a lo largo de un gradiente de condiciones biofísicas y de disturbio; (2) evaluar la descomposición de hojarasca de tres especies arbóreas comunes en la región, que podrían ser incorporadas en distintos momentos de planes de restauración; y (3) establecer una línea de base de variables y especies arbóreas relevantes para la restauración forestal sobre la cual sea posible evaluar su efectividad en años venideros. Este estudio plantea la hipótesis de que la heterogeneidad física y biológica prevalente en la parte alta de la cuenca ocasiona diferencias acentuadas en la acumulación y descomposición de la hojarasca entre varios fragmentos que pueden requerir tratamiento particular, lo cual justificaría la definición de estrategias locales para la restauración de los bosques.

\section{MÉTODOS}

Área de estudio. El estudio se realizó en fragmentos forestales crónicamente perturbados por extracción de madera y leña de seis comunidades de la Sierra Madre de Chiapas, en la parte alta de la cuenca del río Grijalva, particularmente en los territorios de Benito Juárez (BJ), Ejido El Carrizal (EC), Poblado Cambil (PC), Plan Grande (PG) y Vicente Guerrero (VG), ubicadas en el municipio de Motozintla de Mendoza, y Libertad Frontera (LF) en el de Mazapa de Madero (figura 1). Estas localidades colindantes con Guatemala se ubican entre 1.260 y $2.340 \mathrm{~m}$ de altitud, y tienen suelos de tipo acrisol, andosol, cambisol y regosol (INEGI 1988) derivados de rocas ígneas, metamórficas y sedimentarias (Carfantan 1977). Los fragmentos forestales en cada comunidad fueron seleccionados con base en la disposición de sus habitantes para iniciar algunas acciones de restauración en sus terrenos (Gómez-Pineda 2012).

Composición y estructura del bosque. En cada fragmento elegido para la restauración forestal se evaluó la riqueza de especies y la estructura de la vegetación (densidad, área basal) en seis u ocho parcelas circulares de $1.000 \mathrm{~m}^{2}$ (sólo un fragmento con cinco parcelas). Las parcelas se ubicaron con ayuda de una imagen de satélite Google Earth y un GPS para evitar su traslapo y conseguir su distribución en toda la extensión del fragmento, los cuales fueron escasamente mayores a 2-3 ha. Dentro de cada parcela se registró la presencia y se midió el diámetro a la altura del pecho (DAP; 1,30 m de altura) del tronco de todos los individuos (tallos) para obtener la densidad y área basal de cada especie. Los tallos con DAP $>20 \mathrm{~cm}$ se registraron en las parcelas de $1.000 \mathrm{~m}^{2}$. Aquellos con DAP $=11-20 \mathrm{~cm}$ $\mathrm{y}$ con $\mathrm{DAP}=5-10 \mathrm{~cm}$ se registraron en parcelas circulares concéntricas de 500 y $100 \mathrm{~m}^{2}$, respectivamente. Se recolectaron especímenes de las especies de árboles para cote- 


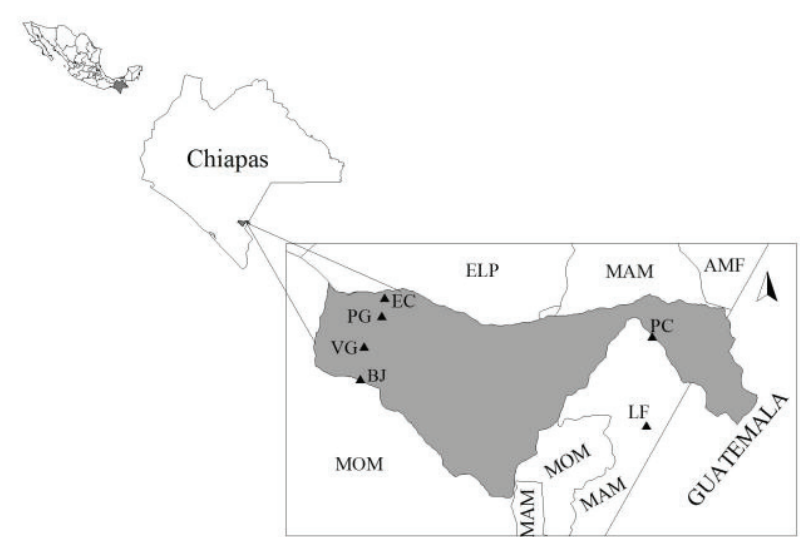

Figura 1. Ubicación de la cuenca en Chiapas, México y de las localidades de estudio: $\mathrm{BJ}=$ Benito Juárez, $\mathrm{EC}=\mathrm{El}$ Carrizal, $\mathrm{LF}=$ Libertad Frontera, $\mathrm{PC}=$ Poblado Cambil, $\mathrm{PG}=$ Plan Grande, $\mathrm{VG}$ $=$ Vicente Guerrero. Se indican también los municipios: AMF= Amatenango de la Frontera, ELP = El Porvenir, MAM = Mazapa de Madero y MOM = Motozintla de Mendoza.

Location of the upper Grijalva River watershed in Chiapas, Mexico, and the studied forest fragments: $\mathrm{BJ}=$ Benito Juárez, $\mathrm{EC}=$ ejido El Carrizal, LF $=$ Libertad Frontera, PC = Poblado Cambil, PG = Plan Grande, $\mathrm{VG}=$ Vicente Guerrero. Municipalities are also indicated: AMF $=$ Amatenango de la Frontera, ELP $=$ El Porvenir, MAM = Mazapa de Madero and $\mathrm{MOM}=$ Motozintla de Mendoza.

jarlas con los depositados en el herbario de El Colegio de la Frontera Sur (ECOSUR). Se obtuvo el área basal (AB) de los tocones presentes en cada parcela, resultantes de la tala acumulada de individuos durante al menos 10 años previos, principalmente para obtener leña; la suma del $\mathrm{AB}$ en cada parcela se consideró como un indicador del grado de disturbio por tala ocurrido en el pasado reciente. Se calculó el valor de importancia relativa (VIR) de cada especie con base en los valores de densidad y dominancia (área basal) relativas; el VIR en cada fragmento se obtuvo con los valores agregados de las parcelas evaluadas en cada localidad.

Variables ambientales. La elevación se estimó con la lectura de tres puntos diferentes en cada parcela con un GPS (eTrex H). La pendiente de las laderas se calculó con un clinómetro Suunto y su exposición respecto al norte (azimut cartográfico) fue leída en una brújula Suunto. La cobertura forestal (o apertura del dosel) se estimó con un densiómetro convexo (Forestry Suppliers) con base en cuatro lecturas en cada parcela.

Muestreo y análisis de suelos. En cada parcela circular de $1.000 \mathrm{~m}^{2}$ se recolectaron tres muestras compuestas de suelo entre 0 y $20 \mathrm{~cm}$ de profundidad (cada una derivada de cinco submuestras separadas $3 \mathrm{~m}$ entre sí). Las muestras fueron secadas al sol, trituradas, tamizadas y llevadas al Laboratorio de Suelos de ECOSUR para determinar: nitrógeno total, (método semi microKjeldhal), fósforo (método de Olsen), capacidad de intercambio catiónico (CIC, método con acetato de amonio $1 \mathrm{~N} \mathrm{y} \mathrm{pH} \mathrm{7),} \mathrm{concen-}$ tración de carbono orgánico (método de Walkley y Black), $\mathrm{pH}$ (relación 1:2 con $\mathrm{H}_{2} \mathrm{O}$ ), capacidad de campo (método de columnas), densidad aparente (método de la probeta) y textura (método de Bouyoucos).

Acumulación de hojarasca. $\mathrm{Al}$ inicio del estudio, en febrero de 2012, se limpió de hojarasca un área de $1 \mathrm{~m}^{2}$ dentro de dos marcos de madera en cada una de las parcelas circulares de $1.000 \mathrm{~m}^{2}$. El efecto de la pendiente sobre la acumulación de hojarasca se evitó con dos terrazas de $50 \times 50 \mathrm{~cm}$ y $0 \%$ de inclinación, labradas en cada parcela circular. La hojarasca acumulada fue evaluada tanto en las superficies inclinadas como en las horizontales de las terrazas después de 280-290 días. La hojarasca recolectada se guardó en bolsas de papel etiquetadas y perforadas y se secó en hornos de aire forzado a $60{ }^{\circ} \mathrm{C}$ hasta peso constante.

Descomposición de hojarasca. Se utilizó el método "litter bag" (Karberg et al. 2008) para evaluar durante 280-290 días, en condiciones de campo, la descomposición de la hojarasca de tres especies comunes en la región (González-Espinosa et al. 2011), con potencial para su uso en la restauración ecológica y cuya hojarasca fuera fácil de conseguir: Alnus acuminata Kunth (dominante en vegetación secundaria temprana derivada del deslizamiento de laderas), Pinus tecunumanii F. Schwerdtf. ex Eguiluz et J.P. Perry y Quercus segoviensis Liebm. Se manejó la hojarasca de las tres especies de manera uniforme en los seis fragmentos estudiados. En cada parcela circular se utilizaron seis bolsas de $20 \times 20 \mathrm{~cm}$ para cada especie, con $10 \mathrm{~g}$ de hojarasca secada previamente (18 bolsas por parcela); las bolsas fueron hechas con malla de polipropileno de $1 \mathrm{~mm}$ de apertura. En el laboratorio se extrajo la hojarasca de cada bolsa con un cepillo y se secó en hornos de aire forzado a $60{ }^{\circ} \mathrm{C}$ hasta peso constante. Se pesó el remanente del material orgánico y se obtuvo la diferencia del peso de la hojarasca al inicio y al final del estudio como medida de la descomposición.

Calidad de hojarasca. Se obtuvieron 10 muestras de hojarasca seca de $30 \mathrm{~g}$ de cada una de las tres especies evaluadas en la descomposición, las cuales se molieron y tamizaron con una malla de $1 \mathrm{~mm}$ de apertura. Se determinó el contenido de nitrógeno (método Kjedahl), carbono, taninos condensables (método de vainillina acidificada) y hemicelulosa, lignina y celulosa (método de van Soest (1994). Los análisis se realizaron en el Laboratorio de Bromatología de ECOSUR.

Análisis estadístico. Se utilizó análisis de varianza (ANDEVA) con un criterio de clasificación para determinar diferencias entre los seis fragmentos en las variables de riqueza de especies y estructura del bosque (área basal, densidad de individuos, cobertura de dosel) con las parcelas circulares 
como repeticiones en cada localidad. Las diferencias entre las variables edáficas se evaluaron con un ANDEVA mixto anidado con dos criterios de clasificación, con tres repeticiones, con fragmentos como factor fijo y las parcelas, anidadas dentro de fragmentos, como factor aleatorio. Para evaluar el efecto de la calidad de las hojas sobre su descomposición, se utilizó un modelo de ANDEVA factorial con dos factores fijos (fragmento y especie) y un factor aleatorio (parcela anidada en fragmento) y seis repeticiones. El efecto del tipo de área de acumulación de la hojarasca (superficie inclinada vs. terrazas horizontales) se evaluó con un ANDEVA factorial mixto anidado con fragmentos y tipo de área de acumulación como factores fijos y parcela anidada dentro de localidad como factor aleatorio, con dos repeticiones. Finalmente, se utilizó un análisis de covarianza con un factor fijo (fragmento) y uno aleatorio (parcela dentro de fragmento), con la pendiente del área de recolección como covariable, para evaluar su efecto sobre la acumulación de la hojarasca sólo en las áreas inclinadas. Los ANDEVA fueron seguidos de pruebas de comparación múltiple de medias de Student-Newman-Keuls $(S-N-K)$. Cuando fue necesario, los valores originales de las variables $(X)$ se transformaron con $Y=\ln (X+1)$ para satisfacer los supuestos del análisis paramétrico; cuando esta condición no se cumplió, se optó por evaluar las diferencias con la prueba de Kruskal-Wallis (Sokal y Rohlf 1995). Se realizó un análisis de componentes principales $(\mathrm{ACP})$ con 11 variables edáficas para reducir la dimensionalidad de la información sobre los suelos (Hair et al. 1999) e identificar un menor número de variables que conjugaran otras posiblemente correlacionadas, a manera de factores. Posteriormente, los valores característicos (eigenvalores) de los dos primeros componentes principales obtenidos fueron ingresados como variables independientes para ajustar un modelo de regresión lineal múltiple (backwards procedure), junto con las variables explicativas que mostraron baja o nula correlación entre sí, para evaluar su efecto sobre la acumulación y descomposición de la hojarasca. Todos los análisis estadísticos se realizaron con el paquete SPSS v. 15.0.

\section{RESULTADOS}

Caracterización de los fragmentos. La riqueza de especies arbóreas, su densidad y área basal difirieron entre los fragmentos estudiados (cuadro 1). Se registró un total de 112 especies leñosas (predominantemente árboles) de 49 familias botánicas (apéndice 1). Las especies con mayor valor de importancia relativa (VIR) fueron Quercus peduncularis Née y Pinus devoniana Lindl. Las localidades con mayor riqueza de especies y mayor área basal fueron BJ y EC (cuadro 1, apéndice 1). Las especies predominantes en BJ fueron Quercus benthamii A. DC. y Clethra chiapensis L.M. González, mientras que en EC fueron Quercus segoviensis Liebm., Q. crassifolia Bonpl. у P. tecunumanii F.Schwerdtf. ex Eguiluz et J.P.Perry. Los fragmentos con doseles más cerrados se encontraron en BJ y EC (cuadro 1).
Características de los suelos. La mayoría de las propiedades edáficas dentro y entre los fragmentos fueron diferentes (materia orgánica, nitrógeno total, capacidad de intercambio catiónico, $\mathrm{pH}$, densidad aparente y capacidad de campo; $P<0,001$ ); el contenido de limo fue diferente entre localidades con $P=0,032$ ). Los contenidos de fósforo, arena y arcilla no difirieron entre fragmentos forestales $(P>0,10)$, pero sí entre las parcelas dentro de ellos $(P<0,001)$. Los mayores valores de materia orgánica, nitrógeno y CIC se encontraron en BJ y EC; por el contrario, en esos mismos fragmentos se registraron los menores valores de densidad aparente (cuadro 2).

Acumulación de hojarasca. Se encontró una interacción entre las localidades y el tipo de superficie de recolección (inclinada u horizontal) de la hojarasca acumulada $\left(F_{5,120}=5,69, P<0,001\right)$, y una diferencia marginal entre las parcelas dentro de las diferentes localidades $\left(F_{36,120}=1,49\right.$, $P=0,057)$. La acumulación de hojarasca sobre superficies inclinadas (50-105\% de pendiente como covariable) fue similar entre localidades $\left(F_{1,41}=0,801 ; P=0,555\right)$ y entre las parcelas dentro de los fragmentos de bosque $\left(F_{36,41}=\right.$ $1,645 ; P=0,062)$. La acumulación de hojarasca sobre superficies horizontales fue diferente entre localidades $\left(F_{5,36}=\right.$ $10,99 ; P<0,001)$, pero no entre las parcelas dentro de los fragmentos de bosque $\left(F_{36,42}=1,15 ; P=0,33\right)$. La acumulación de hojarasca sobre superficies inclinadas varió por un factor de cuatro entre VG $\left(0,09 \pm 0,01 \mathrm{~kg} \mathrm{~m}^{-2}\right)$ y LF $\left(0,35 \pm 0,05 \mathrm{~kg} \mathrm{~m}^{-2}\right)$. La mayor acumulación de hojarasca sobre superficies horizontales se registró también en LF, con un promedio casi tres veces mayor $(1,03 \pm 0,11 \mathrm{~kg}$ $\mathrm{m}^{-2}$ ) al de la acumulación sobre superficies inclinadas en la misma localidad. En el resto de los fragmentos de bosque la acumulación sobre superficies horizontales durante el periodo de evaluación varió entre 0,40 y $0,55 \mathrm{~kg} \mathrm{~m}^{-2}$ (figura 2).

Descomposición de hojarasca. Al cabo de 280-290 días de su exposición a condiciones de campo se encontró una descomposición de la hojarasca de Alnus acuminata de 40$56 \%$, mientras que en Quercus segoviensis y Pinus tecunumanii fue de 30-38 \% y 25-35\%, respectivamente. Aunque se encontraron diferencias entre las especies en cuanto a su descomposición $\left(F_{2,620}=291,44 ; P<0,001\right)$ (figura $3)$, se encontró una interacción con las localidades $\left(F_{10,620}\right.$ $=7,37 ; P<0,001)$. Asimismo, se observaron diferencias en el porcentaje de hojarasca remanente entre las parcelas dentro de los fragmentos en cada localidad $\left(F_{36,620}=3,40\right.$; $P<0,001)$. La descomposición de hojarasca de A. acuminata fue mayor en los fragmentos forestales en BJ, EC y VG $(55,9,56,0$ y $53,0 \%$, respectivamente). La descomposición de $Q$. segoviensis fue relativamente similar entre los seis fragmentos (29,8-38,3\%). Finalmente, la descomposición de $P$. tecunumanii fue más baja (25,1-29,9\%), salvo en PG, donde alcanzó $35 \%$. La descomposición de las tres especies siempre fue menor en PC (figura 3). 
Cuadro 1. Características físicas y estructurales de los seis fragmentos forestales estudiados. $\mathrm{BJ}=\mathrm{Benito}$ Juárez, $\mathrm{EC}=\mathrm{El}$ Carrizal, $\mathrm{LF}=$ Libertad Frontera, $\mathrm{PC}=$ Poblado Cambil, $\mathrm{PG}=$ Plan Grande, $\mathrm{VG}=$ Vicente Guerrero. Medias con distinta letra en la misma fila indican diferencias $(P \leq 0,05$; prueba de $S-N-K)$. Entre paréntesis se presentan para las variables discretas (riqueza de especies) los valores extremos; para las demás variables se indica entre paréntesis \pm un error estándar de la media (EEM). Mayores detalles sobre la composición de especies en los fragmentos se presentan en el apéndice 1.

Physical and forest structure attributes of the six forest fragments studied: $\mathrm{BJ}=$ Benito Juárez, $\mathrm{EC}=\mathrm{El}$ Carrizal, $\mathrm{LF}=$ Libertad Frontera, $\mathrm{PC}=$ Poblado Cambil, $\mathrm{PG}=$ Plan Grande, $\mathrm{VG}=$ Vicente Guerrero. Means within the same line followed by different letters are different $(P \leq 0.05$; $S-N-K$ test). Extreme values of discrete variables (species richness) are shown in parenthesis; for other variables in parentheses are shown \pm 1 standard error of mean (SEM). See appendix 1 for further details on species composition and dominance.

\begin{tabular}{|c|c|c|c|c|c|c|}
\hline Característica & $\mathrm{BJ}$ & $\mathrm{EC}$ & $\mathrm{LF}$ & $\mathrm{PC}$ & PG & VG \\
\hline Número de parcelas evaluadas & 8 & 8 & 8 & 6 & 5 & 6 \\
\hline Latitud $(\mathrm{N})$ & $15^{\circ} 21^{\prime}$ & $15^{\circ} 24^{\prime}$ & $15^{\circ} 20^{\prime}$ & $15^{\circ} 23^{\prime}$ & $15^{\circ} 23^{\prime}$ & $15^{\circ} 22^{\prime}$ \\
\hline Longitud (O) & $92^{\circ} 18^{\prime}$ & $92^{\circ} 17^{\prime}$ & $92^{\circ} 10^{\prime}$ & $92^{\circ} 10^{\prime}$ & $92^{\circ} 18^{\prime}$ & $92^{\circ} 18^{\prime}$ \\
\hline Altitud (m) & $1.992-2.087$ & $1.742-2.338$ & $1.719-1.979$ & $1.259-1.690$ & $1.770-1.828$ & $1.845-2.066$ \\
\hline Pendiente (\%) & $58-97$ & $65-90$ & $56-80$ & $55-90$ & $52-85$ & $60-104$ \\
\hline Cobertura del dosel (\%) & $82,3^{\mathrm{a}}( \pm 3,1)$ & $74,5^{\mathrm{a}}( \pm 5,0)$ & $58,2^{\mathrm{b}}( \pm 3,2)$ & $56,1^{\mathrm{b}}( \pm 6,7)$ & $55,1^{\mathrm{b}}( \pm 4,9)$ & $79,5^{\mathrm{a}}( \pm 2,9)$ \\
\hline \multicolumn{7}{|l|}{ Riqueza por DAP (cm) } \\
\hline $\mathrm{DAP}>20$ & $44^{\mathrm{a}}(2-17)$ & $21^{\mathrm{ab}}(3-8)$ & $4^{c}(1-4)$ & $19^{\mathrm{bc}}(1-8)$ & $6^{\mathrm{bc}}(1-8)$ & $14^{b}(3-7)$ \\
\hline $10<\mathrm{DAP} \leq 20$ & $37^{\mathrm{a}}(1-20)$ & $22^{\mathrm{a}}(1-7)$ & $3^{\mathrm{b}}(0-2)$ & $10^{\mathrm{a}}(1-5)$ & $4^{b}(0-2)$ & $14^{\mathrm{a}}(2-5)$ \\
\hline $5 \leq \mathrm{DAP} \leq 10$ & $35^{\mathrm{ab}}(0-4)$ & $11^{\mathrm{ab}}(0-4)$ & $6^{\mathrm{b}}(0-3)$ & $14^{\mathrm{a}}(0-5)$ & $3^{\mathrm{b}}(0-2)$ & $11^{\mathrm{a}}(1-3)$ \\
\hline \multicolumn{7}{|l|}{ Riqueza por grupo } \\
\hline Coníferas & $4^{\mathrm{ab}}(0-3)$ & $4^{\mathrm{a}}(0-2)$ & $2^{\mathrm{a}}(0-2)$ & $0,0^{\mathrm{b}}$ & $3^{\mathrm{a}}(1-2)$ & $4^{\mathrm{a}}(0-3)$ \\
\hline Latifoliadas & $69^{\mathrm{a}}(9-22)$ & $34^{\mathrm{b}}(2-12)$ & $5^{\mathrm{d}}(1-2)$ & $25^{\mathrm{b}}(3-10)$ & $3^{\mathrm{cd}}(1-2)$ & $17^{\mathrm{bc}}(3-6)$ \\
\hline Total (promedio por ha) & $18^{\mathrm{a}}( \pm 1,5)$ & $8^{b}( \pm 1,1)$ & $3^{c}( \pm 1,1)$ & $6^{\mathrm{b}}( \pm 1,0)$ & $3^{c}( \pm 0,2)$ & $6^{\mathrm{b}}( \pm 0,7)$ \\
\hline \multicolumn{7}{|l|}{ Densidad por DAP $(\mathrm{cm})$} \\
\hline $\mathrm{DAP}>20$ & $216^{\mathrm{a}}( \pm 23)$ & $205^{\mathrm{a}}( \pm 24)$ & $22^{\mathrm{a}}( \pm 23)$ & $116^{\mathrm{b}}( \pm 27)$ & $98^{\mathrm{b}}( \pm 20)$ & $130^{\mathrm{b}}( \pm 20)$ \\
\hline $10<\mathrm{DAP} \geq 20$ & $245^{\mathrm{a}}( \pm 51)$ & $210^{\mathrm{a}}( \pm 42)$ & $160^{\mathrm{ab}}( \pm 48)$ & $374^{\mathrm{a}}( \pm 91)$ & $32^{\mathrm{b}}( \pm 8)$ & $147^{\mathrm{ab}}( \pm 17)$ \\
\hline $5 \leq \mathrm{DAP} \geq 10$ & $862^{\mathrm{a}}( \pm 339)$ & $262^{\mathrm{a}}( \pm 122)$ & $163^{\mathrm{a}}( \pm 84)$ & $557^{\mathrm{a}}( \pm 117)$ & $720^{\mathrm{a}}( \pm 671)$ & $400^{\mathrm{a}}( \pm 121)$ \\
\hline \multicolumn{7}{|l|}{ Densidad por grupo } \\
\hline Coníferas & $106^{\mathrm{b}}( \pm 545)$ & $74,0^{\mathrm{a}}( \pm 29)$ & $145^{\mathrm{a}}( \pm 72)$ & $0^{\mathrm{b}}$ & $744^{\mathrm{a}}( \pm 672)$ & $285^{\mathrm{a}}( \pm 130)$ \\
\hline Latifoliadas & $1.218^{\mathrm{a}}( \pm 303)$ & $603^{\mathrm{ab}}( \pm 141)$ & $398^{b}( \pm 128)$ & $1047^{\mathrm{a}}( \pm 179)$ & $106^{\mathrm{c}}( \pm 40)$ & $392^{\mathrm{b}}( \pm 89)$ \\
\hline Total (individuos $\mathrm{ha}^{-1}$ ) & $1.374^{\mathrm{b}}( \pm 346)$ & $678^{b}( \pm 134)$ & $544^{\mathrm{b}}( \pm 99)$ & $1047^{\mathrm{ab}}( \pm 179)$ & $850^{\mathrm{ab}}( \pm 650)$ & $677^{b}( \pm 106)$ \\
\hline \multicolumn{7}{|l|}{ Área basal $\left(\mathrm{m}^{2} \mathrm{ha}^{-1}\right)$ por DAP $(\mathrm{cm})$} \\
\hline $\mathrm{DAP}>20$ & $32,5^{\mathrm{a}}( \pm 3,8)$ & $22,8^{\mathrm{b}}( \pm 1,8)$ & $18,3^{\text {bcd }}( \pm 3,0)$ & $8,7^{\mathrm{d}}( \pm 2,5)$ & $15,6^{c}( \pm 1,8)$ & $14,8^{\mathrm{cd}}( \pm 1,9)$ \\
\hline $10<\mathrm{DAP} \geq 20$ & $3,9^{\mathrm{a}}( \pm 0,9)$ & $4,1^{\mathrm{a}}( \pm 0,9)$ & $3,2^{\mathrm{a}}( \pm 1,0)$ & $7,2^{\mathrm{a}}( \pm 1,8)$ & $0,5^{\mathrm{b}}( \pm 0,1)$ & $2,4^{\mathrm{a}}( \pm 0,4)$ \\
\hline $5 \leq \mathrm{DAP} \geq 10$ & $3,2^{\mathrm{a}}( \pm 1,3)$ & $1,1^{\mathrm{a}}( \pm 0,5)$ & $0,8^{\mathrm{a}}( \pm 0,5)$ & $2,9^{\mathrm{a}}( \pm 0,6)$ & $3,0^{\mathrm{a}}( \pm 2,7)$ & $1,6^{\mathrm{a}}( \pm 0,4)$ \\
\hline \multicolumn{7}{|l|}{ Área basal por grupo $\left(\mathrm{m}^{2} \mathrm{ha}^{-1}\right)$} \\
\hline Coníferas & $6,1^{\mathrm{ab}}( \pm 4,0)$ & $11,2^{\mathrm{a}}( \pm 4,0)$ & $8,0^{\mathrm{a}}( \pm 2,9)$ & $0,0^{\mathrm{b}}$ & $15,4^{\mathrm{a}}( \pm 1,0)$ & $7,7^{\mathrm{a}}( \pm 3,0)$ \\
\hline Latifoliadas & $33,6^{\mathrm{a}}( \pm 4,8)$ & $16,8^{b}( \pm 3,3)$ & $14,3^{\mathrm{b}}( \pm 2,8)$ & $18,8^{\mathrm{ab}}( \pm 2,3)$ & $3,7^{\mathrm{c}}( \pm 1,0)$ & $10,9^{b}( \pm 2,3)$ \\
\hline Total & $39,7^{\mathrm{a}}( \pm 3,9)$ & $28,0^{\mathrm{b}}( \pm 1,7)$ & $22,4^{c}( \pm 2,4)$ & $18,8^{\mathrm{c}}( \pm 2,3)$ & $19,0^{c}( \pm 1,4)$ & $18,6^{c}( \pm 2,2)$ \\
\hline Área basal tocones (tocones por ha) & $20,3^{\mathrm{a}}( \pm 6,1)$ & $6,2^{\mathrm{a}}( \pm 3,6)$ & $23,8^{\mathrm{a}}( \pm 11,3)$ & $0,28^{\mathrm{a}}( \pm 0,28)$ & $3,9^{\mathrm{a}}( \pm 3,9)$ & $6,1^{\mathrm{a}}( \pm 2,3)$ \\
\hline
\end{tabular}


Cuadro 2. Media ( \pm un error estándar) de variables químicas y físicas del suelo de los seis fragmentos estudiados. BJ = Benito Juárez, $\mathrm{EC}=\mathrm{El}$ Carrizal, $\mathrm{LF}=$ Libertad Frontera, $\mathrm{PC}=$ Poblado Cambil, $\mathrm{PG}=$ Plan Grande, $\mathrm{VG}=$ Vicente Guerrero. Medias con distinta letra en la misma fila indican diferencias $(P \leq 0,05$; prueba de $S-N-K)$.

Mean $( \pm 1$ SEM) of chemical and physical soil attributes in the six forest fragments. BJ $=$ Benito Juárez, EC $=\mathrm{El} \mathrm{Carrizal,} \mathrm{LF}=\mathrm{Libertad}$ Frontera, $\mathrm{PC}=$ Poblado Cambil, $\mathrm{PG}=$ Plan Grande, $\mathrm{VG}=$ Vicente Guerrero. Means within the same line followed by different letters are different $(P \leq 0.05 ; S-N-K$ test $)$.

\begin{tabular}{lrrrrrr}
\hline \multicolumn{1}{c}{ Variable } & \multicolumn{1}{c}{$\mathrm{BJ}$} & $\mathrm{EC}$ & $\mathrm{LF}$ & $\mathrm{PC}$ & \multicolumn{1}{c}{ PG } & VG \\
\hline Materia orgánica (\%) & $6,80^{\mathrm{a}}(0,60)$ & $7,68^{\mathrm{a}}(0,57)$ & $4,06^{\mathrm{b}}(0,28)$ & $3,86^{\mathrm{b}}(0,87)$ & $3,45^{\mathrm{b}}(0,34)$ & $5,60^{\mathrm{ab}}(1,00)$ \\
Nitrógeno total (\%) & $0,39^{\mathrm{a}}(0,03)$ & $0,46^{\mathrm{a}}(0,03)$ & $0,20^{\mathrm{b}}(0,01)$ & $0,22^{\mathrm{b}}(0,05)$ & $0,21^{\mathrm{b}}(0,03)$ & $0,32^{\mathrm{ab}}(0,06)$ \\
Relación C/N & $10,10^{\mathrm{c}}(0,02)$ & $9,59^{\mathrm{d}}(0,18)$ & $11,74^{\mathrm{a}}(0,23)$ & $10,55^{\mathrm{b}}(0,09)$ & $9,66^{\mathrm{c} d}(0,26)$ & $10,04^{\mathrm{c}}(0,03)$ \\
Fósforo (mg kg-1) & $5,10^{\mathrm{a}}(1,12)$ & $15,92^{\mathrm{a}}(5,18)$ & $14,11^{\mathrm{a}}(2,97)$ & $5,97^{\mathrm{a}}(1,67)$ & $10,83^{\mathrm{a}}(2,57)$ & $9,79^{\mathrm{a}}(2,43)$ \\
CIC (cmol kg- $)$ & $24,96^{\mathrm{ab}}(1,38)$ & $24,90^{\mathrm{a}}(0,66)$ & $20,32^{\mathrm{bc}}(1,07)$ & $17,94^{\mathrm{c}}(0,91)$ & $17,54^{\mathrm{c}}(1,51)$ & $18,96^{\mathrm{bc}}(1,66)$ \\
pH & $5,66^{\mathrm{c}}(0,06)$ & $6,06^{\mathrm{b}}(0,09)$ & $5,46^{\mathrm{c}}(0,13)$ & $7,10^{\mathrm{a}}(0,11)$ & $6,36^{\mathrm{ab}}(0,15)$ & $5,51^{\mathrm{c}}(0,08)$ \\
Densidad aparente $\left.(\mathrm{g} \mathrm{mL})^{-1}\right)$ & $1,02^{\mathrm{c}}(0,03)$ & $1,10^{\mathrm{b}}(0,02)$ & $1,17^{\mathrm{a}}(0,03)$ & $1,21^{\mathrm{ab}}(0,04)$ & $1,22^{\mathrm{ab}}(0,04)$ & $1,29^{\mathrm{ab}}(0,07)$ \\
Capacidad de campo (\%) & $47,99^{\mathrm{a}}(0,46)$ & $37,23^{\mathrm{b}}(1,56)$ & $34,03^{\mathrm{b}}(1,16)$ & $35,54^{\mathrm{b}}(2,82)$ & $34,80^{\mathrm{b}}(1,99)$ & $37,40^{\mathrm{b}}(1,00)$ \\
Arena (\%) & $41,78^{\mathrm{a}}(2,41)$ & $44,94^{\mathrm{a}}(3,77)$ & $49,80^{\mathrm{a}}(1,23)$ & $39,49^{\mathrm{a}}(4,61)$ & $40,30^{\mathrm{a}}(7,66)$ & $50,16^{\mathrm{a}}(2,31)$ \\
Limo (\%) & $44,16^{\mathrm{a}}(2,71)$ & $37,92^{\mathrm{ab}}(3,05)$ & $31,58^{\mathrm{b}}(1,73)$ & $44,66^{\mathrm{ab}}(3,65)$ & $41,46^{\mathrm{ab}}(6,91)$ & $34,66^{\mathrm{b}}(1,56)$ \\
Arcilla (\%) & $14,05^{\mathrm{a}}(1,00)$ & $17,15^{\mathrm{a}}(1,21)$ & $18,62^{\mathrm{a}}(1,22)$ & $15,84^{\mathrm{a}}(1,45)$ & $18,23^{\mathrm{a}}(2,40)$ & $15,17^{\mathrm{a}}(1,31)$ \\
\hline
\end{tabular}

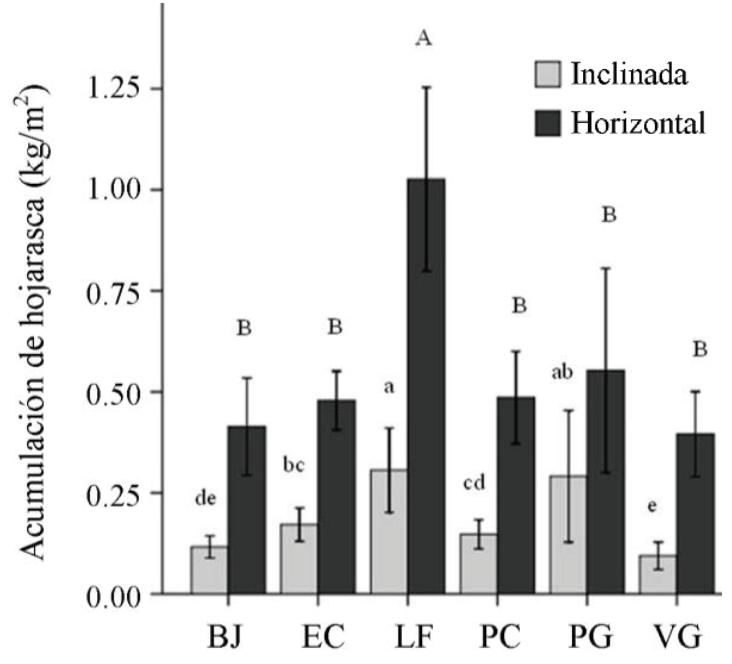

Figura 2. Acumulación de hojarasca promedio $( \pm$ el intervalo de confianza de $95 \%$ ) en superficies horizontales $(0 \%)$ e inclinadas en seis fragmentos forestales. Letras minúsculas distintas indican diferencias $(P \leq 0,05)$ en la acumulación sobre superficies inclinadas; letras mayúsculas distintas indican diferencias $(P \leq 0,05)$ en la acumulación sobre superficies horizontales con la prueba de $S-N-K$.

Litter accumulation (mean $\pm 95 \%$ confidence interval) on horizontal and tilted collecting surfaces in six forest fragments. Lowercase letters indicate litter accumulation differences $(P \leq 0.05)$ on tilted surfaces; uppercase letters indicate litter accumulation differences $(P \leq 0.05)$ on horizontal surfaces $(S-N-K$ test $)$.
Atributos químicos de las hojas. La concentración de nitrógeno y hemicelulosa ( $P<0,001$ en ambos casos) fueron mayores en $A$. acuminata que en $Q$. segoviensis y $P$. tecunumanii, en tanto que la concentración de celulosa, taninos condensables, la relación $\mathrm{C} / \mathrm{N}$ y la relación lignina/nitrógeno presentaron mayores valores en las hojas de $P$. tecunumanii $(P<0,001$ en todos los casos). La concentración de carbono no fue diferente en las hojas de las tres especies (cuadro 3).

Determinantes de la acumulación de hojarasca. El análisis de componentes principales realizado sobre las 11 variables edáficas identificó dos componentes que en conjunto explicaron $52 \%$ de la varianza total (cuadro 4). El primer componente incluyó, de manera predominante, al contenido de nitrógeno $(0,923)$, el contenido de materia orgánica $(0,916)$ y la capacidad de intercambio catiónico $(0,677) \mathrm{y}$, en menor grado, a las variables físicas densidad aparente $(-0,809)$ y capacidad de campo $(0,671)$. De manera diferente, en el segundo componente las variables químicas tuvieron pesos considerablemente menores $(<0,32)$ y fueron las variables físicas determinantes de la textura y relacionadas con la capacidad de retención de agua las que tuvieron el mayor peso: contenido de arena $(-0,948)$ y limo $(0,872)$ (cuadro 4). El modelo que mejor se ajustó a la acumulación de hojarasca sobre superficies inclinadas explicó $28 \%$ de la varianza total e incluyó la densidad arbórea $(t=-2,648 ; P=0,012)$, la orientación de la ladera 


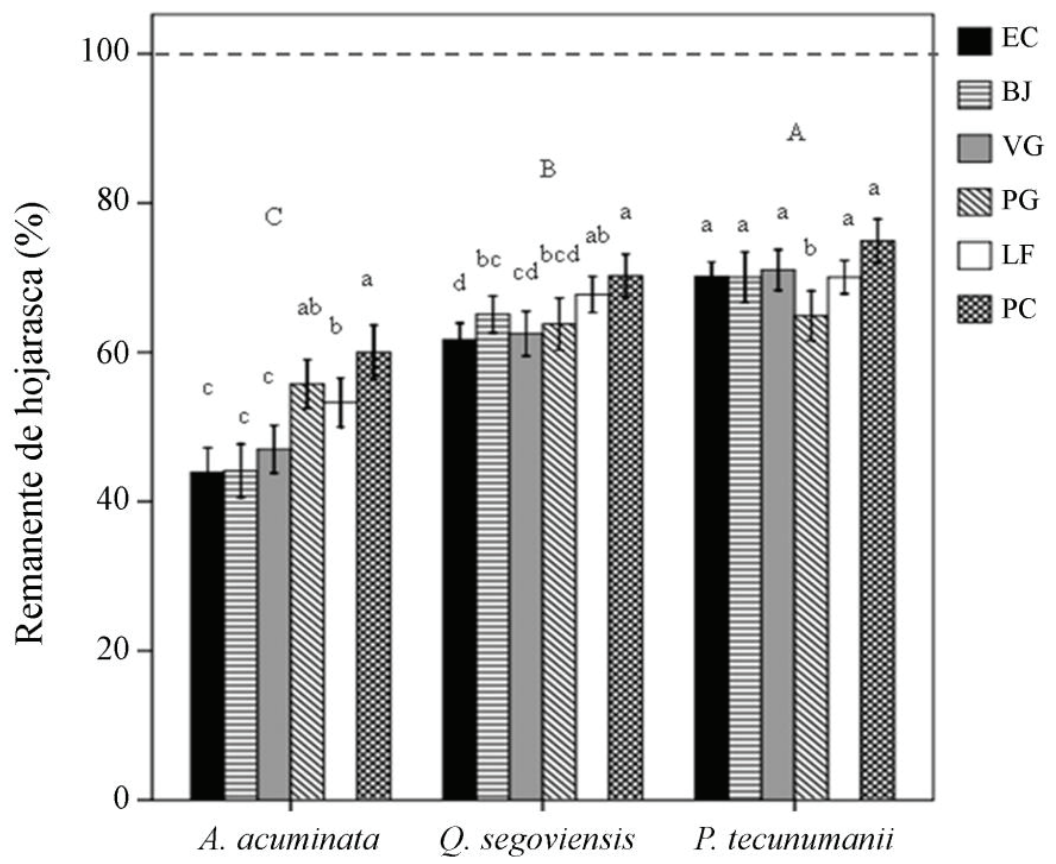

Figura 3. Porcentaje promedio de peso remanente de hojarasca ( \pm el intervalo de confianza de $95 \%$ ) resultante de la descomposición de tres especies en seis fragmentos forestales. Letras minúsculas entre las barras dentro de las especies indican diferencias entre localidades; letras mayúsculas indican diferencias $(P \leq 0,05)$ entre especies con la prueba de $S-N-K$.

Remaining weight of decomposing litter (mean percentage $\pm 95 \%$ confidence interval) in six forest fragments. Lowercase letters among bars within species indicate differences among forest fragments; uppercase letters indicate differences among species $(P \leq 0,05$; $S$ - $N$ - $K$ test).

Cuadro 3. Medias ( \pm 1 EEM) de atributos químicos de hojas de tres especies utilizadas para estimar descomposición de hojarasca. Medias con distinta letra en la misma columna son diferentes $(P \leq 0,05)$ con la prueba de $S-N-K$.

Means ( \pm 1 SEM) of the chemical attributes of litter of the three species used to estimate litter decomposition. Means within the same line followed by different letters are different $(P \leq 0.05 ; S-N-K$ test).

\begin{tabular}{lcccccccc}
\hline Especies & $\begin{array}{c}\mathrm{C} \\
(\%)\end{array}$ & $\begin{array}{c}\mathrm{N} \\
(\%)\end{array}$ & $\begin{array}{c}\text { Lignina } \\
(\%)\end{array}$ & $\begin{array}{c}\text { Celulosa } \\
(\%)\end{array}$ & $\begin{array}{c}\text { Hemicelulosa } \\
(\%)\end{array}$ & $\begin{array}{c}\text { Taninos } \\
\left(\mathrm{mg} \mathrm{kg}^{-1}\right)\end{array}$ & $\begin{array}{c}\text { Relación } \\
\mathrm{C} / \mathrm{N}\end{array}$ & $\begin{array}{c}\text { Relación lignina/ } \\
\text { nitrógeno }\end{array}$ \\
\hline Alnus & $45,05^{\mathrm{a}}$ & $1,57^{\mathrm{a}}$ & $25,34^{\mathrm{b}}$ & $4,11^{\mathrm{b}}$ & $3,93^{\mathrm{a}}$ & $0,05^{\mathrm{c}}$ & $28,81^{\mathrm{b}}$ & $16,23^{\mathrm{c}}$ \\
acuminata & $(0,62)$ & $(0,03)$ & $(0,53)$ & $(0,43)$ & $(0,43)$ & $(0,00)$ & $(0,61)$ & $(0,53)$ \\
& & & & & & & & \\
Pinus & $44,32^{\mathrm{a}}$ & $0,61^{\mathrm{b}}$ & $40,45^{\mathrm{a}}$ & $23,94^{\mathrm{a}}$ & $0,86^{\mathrm{c}}$ & $0,54^{\mathrm{a}}$ & $76,03^{\mathrm{a}}$ & $69,21^{\mathrm{a}}$ \\
tecunumanii & $(0,42)$ & $(0,05)$ & $(0,26)$ & $(0,51)$ & $(0,07)$ & $(0,01)$ & $(5,45)$ & $(4,71)$ \\
Quercus & $43,35^{\mathrm{a}}$ & $0,68^{\mathrm{b}}$ & $35,78^{\mathrm{a}}$ & $15,97^{\mathrm{c}}$ & $1,46^{\mathrm{b}}$ & $0,13^{\mathrm{b}}$ & $64,61^{\mathrm{a}}$ & $53,14^{\mathrm{b}}$ \\
segoviensis & $(0,38)$ & $(0,02)$ & $(1,49)$ & $(0,33)$ & $(0,22)$ & $(0,01)$ & $(1,83)$ & $(53,14)$ \\
\hline
\end{tabular}

( $t=2,156 ; P=0,016)$ y el primer componente principal (más relacionado con la fertilidad del suelo) $(t=-2,285$; $P=0,028)$. Es decir, la acumulación de hojarasca fue mayor cuando la orientación del fragmento tuvo valores cercanos al suroeste en fragmentos poco densos sobre suelos de baja fertilidad. La variación en la acumulación de hojarasca sobre superficies horizontales se explicó hasta $39 \%$ con el modelo de regresión. Los factores que determinaron la acumulación de hojarasca sobre la superficie horizontal fueron el primer componente principal $(t=-3,529$;
$P=0,001)$, el área basal $(t=3,240 ; P=0,003)$, la orientación de la ladera $(t=2,815 ; P=0,008)$ y la densidad arbórea $(t=-2,774 ; P=0,009)$. Esto es, la mayor acumulación de hojarasca se asoció con la orientación de la ladera hacia el suroeste en rodales con mayor área basal, pero con baja densidad y baja fertilidad del suelo.

Determinantes de la descomposición de la hojarasca. Los factores que determinaron la descomposición dependieron de la especie. El modelo ajustado para explicar la descom- 
Cuadro 4. Varianza acumulada, valores característicos (eigenvalores) y peso de 11 variables químicas y físicas del suelo, concentradas en los dos primeros componentes principales que permiten reducir la dimensionalidad de la información edáfica en sólo dos factores.

Accumulated variance, eigenvalues and loads of 11 chemical and physical soil variables included a principal component analysis aimed at reducing the dimensionality of soil attributes into only two factors.

\begin{tabular}{lccc}
\hline \multirow{2}{*}{ Característica } & \multicolumn{2}{c}{ Cargas de los factores extraídos } & \multirow{2}{*}{ Comunalidades } \\
\cline { 2 - 3 } & Factor 1 & Factor 2 & - \\
Varianza explicada (\%) & 32,978 & 19,424 & - \\
Valor característico (eigenvalor) & 3,628 & 2,137 & \\
Variables introducidas & & & 0,616 \\
$\quad$ Fósforo & $-0,013$ & 0,316 & 0,944 \\
Nitrógeno & 0,923 & $-0,191$ & 0,928 \\
Materia orgánica & 0,916 & $-0,251$ & 0,588 \\
Relación C/N & $-0,360$ & $-0,310$ & 0,729 \\
CIC & 0,677 & $-0,143$ & 0,337 \\
pH & $-0,175$ & 0,297 & 0,684 \\
Densidad aparente & $-0,809$ & 0,018 & 0,676 \\
Capacidad de campo & 0,671 & 0,088 & 0,979 \\
Arena & $-0,205$ & $-0,948$ & 0,935 \\
Limo & 0,318 & 0,872 & 0,742 \\
Arcilla & $-0,265$ & 0,256 & \\
\hline
\end{tabular}

posición de $A$. acuminata explicó $0,62 \%$ de la variación total y los factores que contribuyeron fueron la cobertura del dosel $(t=4,77 ; P<0,001)$, la altitud $(t=3,20$; $P=0,003)$ y la orientación $(t=2,29 ; P=0,028)$. Esto es, A. acuminata se descompuso con mayor rapidez en laderas altas de orientación SO con bosques cerrados. El modelo con un mejor ajuste para la descomposición de $Q$. segoviensis explicó una proporción de la varianza total considerablemente menor $\left(R^{2}=0,19\right)$ y los factores explicativos fueron la altitud $(t=3,10 ; P=0,004)$ y el componente principal relacionado con retención de humedad en el suelo $(t=2,10 ; P<0,046)$, lo que indica una mayor descomposición en sitios altos con suelos que retienen mayor humedad. En el caso de P. tecunumanii, el modelo que mejor explicó la descomposición de hojarasca tuvo también un valor bajo del coeficiente de determinación $\left(R^{2}=0,17\right) \mathrm{y}$ sólo dependió de la altitud $(t=3,143 ; P=0,003)$.

\section{DISCUSIÓN}

Acumulación de hojarasca. Las técnicas descritas en la literatura para evaluar acumulación de hojarasca son muy diferentes, lo cual complica la comparación de sus resultados. Como en otros estudios, las diferencias en la acumulación de la hojarasca observadas pueden explicarse por las condiciones de cada fragmento o localidad (Vitousek et al. 1994, Sariyildiz y Küçük 2008). En nuestro estudio, la mayor acumulación de hojarasca se encontró en
LF $\left(0,38 \mathrm{Mg} \mathrm{ha}^{-1} \mathrm{mes}^{-1}\right)$, una acumulación casi dos veces mayor a la de un bosque en restauración del Parque Nacional Lagunas de Montebello (PNLM), Chiapas $(0,20 \mathrm{Mg}$ $\mathrm{ha}^{-1} \mathrm{mes}^{-1}$ ), pero inferior a la observada en asociaciones más maduras dentro del mismo parque: bosque de Pinus spp. $\left(0,43 \mathrm{Mg} \mathrm{ha}^{-1} \mathrm{mes}^{-1}\right)$, bosque de especies de Pinus-QuercusLiquidambar $\left(0,44 \mathrm{Mg} \mathrm{ha}^{-1} \mathrm{mes}^{-1}\right)$, bosque de Quercus spp. $\left(0,54 \mathrm{Mg} \mathrm{ha}^{-1} \mathrm{mes}^{-1}\right)$ y bosque de Pinus-Quercus $(0,63 \mathrm{Mg}$ ha $^{-1}$ mes $^{-1}$ ) (Rocha-Loredo y Ramírez-Marcial 2009), así como a la de un bosque de niebla de Veracruz, México $\left(0,71 \mathrm{Mg} \mathrm{ha}^{-1} \mathrm{mes}^{-1}\right)$ (Williams-Linera y Tolome 1996). En el área de estudio, las especies de Pinus y la mayoría de Quercus spp. se encuentran en sitios relativamente pobres, con suelos poco fértiles, con baja capacidad de campo y bajos valores de $\mathrm{pH}$ que también reducen la descomposición de la hojarasca y así podrían permitir su mayor acumulación (Gaspar-Santos 2013). Aunque no se ha evaluado de manera directa, la interacción entre la velocidad de los vientos y las pendientes más abruptas en nuestra área de estudio (a menudo $>45^{\circ}$ ) respecto a las laderas del PNLM, aunado al aclareo crónico al que han estado sometidos los fragmentos forestales estudiados, pueden ayudar a explicar los menores niveles de acumulación de hojarasca observados.

La acumulación de la hojarasca sobre superficies inclinadas fue afectada de manera negativa por la densidad arbórea y la fertilidad del suelo, pero fue favorecida por la orientación de las parcelas hacia el suroeste. Sobre superficies horizontales, además de la baja densidad arbórea, 
la orientación de las parcelas hacia el suroeste y la baja fertilidad del suelo, la acumulación fue favorecida por una mayor área basal. Williams-Linera y Tolome (1996) encontraron una relación positiva entre la acumulación de hojarasca y el área basal de las especies dominantes (Liquidambar macrophylla Oerst. y Quercus spp.) en un bosque de niebla del centro de Veracruz. Los resultados obtenidos en este estudio son coincidentes y es posible explicar la mayor acumulación encontrada en LF, localidad en la que Pinus devoniana y Quercus peduncularis mostraron mayor área basal. Posiblemente, la relación inversa de la densidad arbórea con la acumulación de hojarasca se deba a la presencia de árboles de gran talla, que pueden producir gran cantidad de hojarasca en sitios con densidad relativamente baja. Sin embargo, es necesario realizar estudios más detallados sobre las relaciones entre la estructura forestal, los atributos físicos del sitio y la acumulación de hojarasca, así como contar con un mayor número de muestras horizontales de captación de hojarasca de mayores dimensiones a las usadas en este estudio.

La acumulación de hojarasca y la orientación de la ladera pueden estar relacionadas, pero en función de las condiciones regionales en cuanto a latitud, la posición de las cadenas montañosas y las rutas de los vientos húmedos. Por ejemplo, Sariyildiz y Küçkü (2008) encontraron una mayor acumulación de hojarasca en laderas orientadas al norte en bosques caducifolios del noreste de Turquía. En este estudio se encontró una mayor acumulación en las laderas orientadas hacia al suroeste. Por una parte, las laderas orientadas al sur tienen una mayor exposición al sol, que puede causar mayor déficit hídrico, con un efecto más pronunciado en latitudes medias (Holland y Steyn 1975), con consecuencias negativas en la retención de humedad del suelo, la actividad de los microorganismos y, a la postre, la descomposición de la hojarasca (Sariyildiz 2008). Por otra parte, la materia orgánica incrementa la retención de humedad y el aporte de nutrimentos (Lavelle et al. 1993), y se ha señalado que los suelos con escasa materia orgánica o baja fertilidad pueden acentuar el estrés hídrico y conducir a una mayor abscisión de hojas, sobre todo en especies caducifolias, y así aumentar la acumulación de hojarasca (Martínez-Yrízar y Sarukhán 1990, Kumar y Deepu 1992).

Descomposición de hojarasca. Los valores de descomposición encontrados en este estudio (40-56 \% en $\mathrm{A}$. acuminata, 30-38 \% en Quercus segoviensis y $25-35 \%$ en Pinus tecunumanii) son menores a los encontrados en bosques de montaña de la meseta central de Chiapas (México) (Rocha-Loredo y Ramírez-Marcial 2009), quienes al cabo de un lapso similar (300 días) encontraron que la descomposición de hojarasca de $A$. acuminata fue de $61-67 \%$ y de 34-52 \% en la de Chiranthodendron pentadactylon Larreat., Clethra suaveolens Turcz., Liquidambar styraciflua L., Pinus oocarpa Schiede ex Schltdl, Quercus crassifolia Benth. y $Q$. sapotifolia Liebm. En ambos estudios el orden de la descomposición fue A. acuminata $>$ Quercus spp. $>$
Pinus spp., lo que sugiere la influencia de la calidad de la hojarasca en el proceso de descomposición en diferentes condiciones ambientales.

La hojarasca con menor descomposición ( $Q$. segoviensis y $P$. tecunumanii) fue aquella con mayor concentración de compuestos recalcitrantes, como celulosa, lignina y taninos condensables, y que también tuvo una alta relación de carbono/nitrógeno y lignina/nitrógeno. Esto es consistente con estudios en los que la descomposición se correlaciona inversamente con la concentración de taninos condensables, lignina, una relación alta carbono/nitrógeno y de lignina/nitrógeno (Kumar y Deepu 1992, SchererLorenzen et al. 2007, Rocha-Loredo y Ramírez-Marcial 2009) y, de manera directa, con una alta concentración de nitrógeno (Xuluc-Tolosa et al. 2003).

La descomposición de hojarasca de A. acuminata se relaciona con una mayor cobertura de dosel, la mayor altitud y la orientación de las parcelas hacia el suroeste. La cobertura del dosel induce y modera cambios ambientales locales, especialmente en la humedad y fauna del suelo, con efectos positivos sobre la descomposición (von Arx et al. 2012). Sariyildiz (2008) registró una mayor descomposición de la hojarasca de tres especies en la medida en que los claros en bosques de Turquía eran menores $(<15 \mathrm{~m}$ de diámetro). Vitousek et al. (1994) encontraron en Hawaii mayor descomposición de hojarasca de Metrosideros polymorpha Gaudich. en sitios húmedos a menor altitud, a lo largo de un gradiente altitudinal y en tres sustratos diferentes. Posiblemente, y no obstante la ocurrencia de menores temperaturas, el resultado obtenido en este estudio se debe al aumento de la humedad en los sitios de mayor altitud. La mayor descomposición de hojarasca en las laderas expuestas al suroeste puede deberse a la presencia de mayores temperaturas dada la mayor cantidad de horas de luz recibidas (Holland y Steyn 1975).

Implicaciones para la restauración. La descomposición de la hojarasca es un proceso ecosistémico clave del ciclo de nutrimentos. En un plan de restauración forestal se requiere identificar las especies arbóreas nativas que aporten más materia orgánica y favorezcan el retorno de nutrimentos al suelo mediante la acumulación y descomposición de la hojarasca. El plan de restauración debe considerar, además de las condiciones biofísicas del sitio, las necesidades de las comunidades propietarias del territorio (Chazdon 2008). Con base en los resultados de este estudio, se puede proponer el uso de $A$. acuminata al inicio de la restauración de laderas deforestadas debido a la alta tasa de descomposición de su hojarasca, además de su capacidad de fijación de nitrógeno (Sharma y Ambasht 1987). La facilitación propiciada por A. acuminata podría ser aprovechada de manera simultánea, o en secuencia, con la introducción de Quercus spp., productores de alta cantidad de hojarasca y que forman doseles cerrados bajo los cuales pueden establecerse especies nativas tolerantes a la sombra (Ramírez-Marcial et al. 2010). 


\section{CONCLUSIONES}

Las diferencias en la composición y estructura de la vegetación y de la fertilidad del suelo de los fragmentos de bosque estudiados determinan la variación local en los procesos de acumulación y descomposición de la hojarasca. La descomposición de la hojarasca de tres especies estudiadas varía en función de sus atributos químicos en el orden A. acuminata $>Q$. segoviensis $>P$. tecunumanii y se relaciona con la cobertura del dosel, la altitud y la exposición de las laderas hacia al suroeste. La acumulación es mayor en los fragmentos con escasa fertilidad del suelo, baja humedad y que reciben mayor cantidad de luz, factores que mantienen condiciones de estrés hídrico y restringen la descomposición de la hojarasca. Los resultados sugieren la conveniencia de concentrar los esfuerzos iniciales de restauración con plantaciones de juveniles de Alnus acuminata, seguidos de Quercus segoviensis, por su aportación de materia orgánica y nutrimentos a través de la descomposición de su hojarasca y su capacidad de fijación simbiótica de nitrógeno.

\section{AGRADECIMIENTOS}

Agradecemos a Henry E. Castañeda Ocaña, Soledad Gómez Gómez, Zenaida Hernández López, Alfonso Luna Gómez, Miguel Martínez Icó, Eliana Noguera Savelli, Olmar Sántiz López y Abel de J. Roblero Vázquez por su apoyo en el trabajo de campo. Emmanuel Valencia Barrera y Karim Musálem Castillejos ayudaron con el SIG, Guadalupe Pérez, Miguel Ángel López y J. Jesús Carmona con los análisis de laboratorio de suelos y hojas y Érika Gómez Pineda y J. Anahí Hernández García en el contacto con las comunidades. Deseamos agradecer a las numerosas personas de las comunidades por su hospitalidad y apoyo en diferentes actividades del proyecto. Alejandro Morón Ríos, María Mercedes Castillo Uzcanga, Miguel Ángel Castillo Santiago, y en especial Sandra Chediack ofrecieron valiosos comentarios. Este trabajo ha sido posible gracias al apoyo del Fondo Institucional de Fomento Regional para el Desarrollo Científico, Tecnológico y de Innovación (FORDECyT) del Consejo Nacional de Ciencia y Tecnología (CONACyT) a través del convenio 143303: "Gestión y estrategias de manejo sustentable para el desarrollo regional en la cuenca hidrográfica transfronteriza Grijalva", apoyado por fondos concurrentes de la entonces Secretaría de Recursos Naturales y Protección Ambiental (SERNAPAM) del estado de Tabasco. ESGS agradece al CONACyT por una beca $(386235 / 255215)$ otorgada para la realización de sus estudios de maestría en ciencias.

\section{REFERENCIAS}

Bens O, NA Wahl, H Fischer, RF Hüttl. 2007. Water infiltration and hydraulic conductivity in sandy cambisols: impacts of forest transformation on soil hydrological properties. Eu- ropean Journal of Forest Research 126: 101-109. DOI: 10.1007/s10342-006-0133-7.

Berg B, C McClaugherty. 2008. Plant litter: decomposition, humus formation, carbon sequestration. Berlín, Alemania. Springer. $338 \mathrm{p}$.

Bradshaw CJ, NS Sodhi, KSH Peh, BW Brook. 2007. Global evidence that deforestation amplifies flood risk and severity in the developing world. Global Change Biology 13: 23792395. DOI: 10.1111/j.1365-2486.2007.01446.x.

Carfantan JC. 1977. La cobijadura de Motozintla-un paleoarco volcánico en Chiapas, México. Revista Mexicana de Ciencias Geológicas 1(2): 133-137.

Chazdon RL. 2008. Beyond deforestation: Restoring forest and ecosystem services on degraded lands. Science 320: 14581460. DOI: $10.1126 /$ science. 1155365.

CIFOR (Center for International Forestry Research, ID). 2005. Forests and floods: drowning in fiction or thriving on facts? Sindang Barang, Indonesia. FAO - CIFOR. 20 p.

Gaspar-Santos ES. 2013. Determinantes de la acumulación y descomposición de la hojarasca en bosques de la cuenca alta del rio Grijalva, Chiapas, México. Tesis de maestría en ciencias. San Cristóbal de las Casas, Chiapas, México. El Colegio de la Frontera Sur. 66 p.

Gómez-Pineda, E. 2012. Estrategias para la restauración forestal en comunidades del municipio de Motozintla, Chiapas, México. Tesis de Maestría en ciencias. San Cristóbal de las Casas, Chiapas, México. El Colegio de la Frontera Sur. 46 p.

González-Espinosa, M, JA Meave, FG Lorea-Hernández, G Ibarra-Manríquez, AC Newton, eds. 2011. The Red List of Mexican cloud forest trees. Cambridge, Reino Unido, Flora and Fauna International. 149 p.

Hair JF Jr, RE Anderson, RL Tatham, WC Black. 1999. Análisis multivariante. Madrid, España, Prentice Hall Iberia. 799 p.

Hamilton L. 1995. Mountain cloud forest conservation and research: A synopsis. Mountain Research and Development 15: 259-266.

Holland PG, DG Steyn. 1975. Vegetational responses to latitudinal variations in slope angle and aspect. Journal of Biogeography 2: 179-183.

INEGI (Instituto Nacional de Estadística y Geografía, MX). 1988. Carta temática y edafológica 1:250 000. Información digital en formato shp; LAIGE-ECOSUR. San Cristóbal de Las Casas, Chiapas, México.

Islam KR, MR Ahmed, MK Bhuiyan, A Badruddin. 2001. Deforestation effects on vegetative regeneration and soil quality in tropical semi-evergreen degraded and protected forest of Bangladesh. Land Degradation \& Development 12: 45-56.

Karberg NJ, NA Scott y CP Giardina. 2008. Methods for estimating litter decomposition. In Hoover CM ed. Field measurement for forest carbon monitoring. Nueva York, NY, USA. Springer. 103-111.

Kumar BM, JK Deepu. 1992. Litter production and decomposition dynamics in moist deciduous forests of the western Ghats in Peninsular India. Forest Ecology and Management 50(30): 181-201.

Lara A, C Little, R Urrutia, J McPhee, C Álvarez-Garretón, C. Oyarzún, D Soto, P. Donoso, L Nahuelhual, M Pino, I Arismendi. 2009. Assessment of ecosystem services as an opportunity for the conservation and management of native forests in Chile. Forest Ecology and Management 258(4): 415-429. 
Laurance FW. 2007. Forest and floods. Nature 449: 409-410.

Lavelle P, E Blanchart, A Martin, S Martin, A Spain. 1993. A hierarchical model for decomposition in terrestrial ecosystems: Application to soils of the humid tropics. Biotropica 25(2): 130-150.

Martínez-Yrízar A, J Sarukhán. 1990. Litterfall patterns in a tropical deciduous forest in Mexico over a five- year period. Journal of Tropical Ecology 6(4): 433-444.

Ramírez-Marcial N, M González-Espinosa, A Camacho-Cruz, D Ortiz-Aguilar. 2010. Forest restoration in Lagunas de Montebello National Park, Chiapas, Mexico. Ecological Restoration 28(3): 354-360.

Restrepo C, RW Lawrence, BS Aaron, B Rainer. 2009. Landsliding and its multiscale influence on mountain escapes. BioScience 59(8): 685-698. DOI: 10.1525/bio.2009.59.8.10.

Richter M. 2000. The ecological crisis in Chiapas: a case study from Central America. Mountain Research and Development 20(4): 332-339.

Rocha-Loredo AG, N Ramírez-Marcial. 2009. Producción y descomposición de hojarasca en diferentes condiciones sucesionales del bosque de pino-encino en Chiapas, México. Boletín de la Sociedad Botánica de México 84: 1-12.

Sariyildiz T. 2008. Effects of gap-size classes on long-term litter decomposition rates of beech, oak and chestnut species at high elevations in northeast Turkey. Ecosystems 11(6): 841853. DOI: 10.1007/s10021-008-9164-x.

Sariyildiz T, M Küçkü. 2008. Litter mass in deciduous and coniferous trees in Artvin, northeast Turkey: Relationships with litter quality, microclimate, and soil characteristics. Turkish Journal of Agriculture and Forestry 37(9): 547-559.

Scherer-Lorenzen M, JL Bonilla, C Potvin. 2007. Tree species richness affects litter production and decomposition rates in a tropical biodiversity experiment. Oikos 116(12): 21082124. DOI: 10.1111/j.2007.0030-1299.16065.x.

Sharma E, RS Ambasht. 1987. Litterfall, decomposition and nutrient release in an age sequence of Alnus nepalensis plantation stands in the eastern Himalaya. Journal of Ecology 75: 997-1010.

Sokal R, JF Rohlf. 1995. Biometry: the principles and practice of statistics in biological research. New York, NY, USA. Freeman. 887 p.

van Soest PJ. 1994. Nutritional ecology of the ruminant. Ithaca, NY, USA. Cornell University Press. 476 p.

Vitousek PM, RL Sanford. 1986. Nutrient cycling moist tropical forest. Annual Review of Ecology and Systematics 17: 137-67.

Vitousek PM, DR Turner, WJ Parton, RL Sanford. 1994. Litter decomposition on the Mauna Loa environmental matrix, Hawaii: Patterns, mechanisms and models. Ecology 75(2): 418-429.

von Arx G, M Dobbertin, M Rebetez. 2012. Spatio-temporal effects of forest canopy on understory microclimate in a long-term experiment in Switzerland. Agricultural and Forest Meteorology 167: 144-155.

Williams-Linera G, J Tolome. 1996. Litterfall, temperate and tropical dominant trees, and climate in a Mexican lower montane forest. Biotropica 28(4): 649-656.

Xuluc-Tolosa FJ, HFM Vester, N Ramírez-Marcial, J Castellanos-Albores, D Lawrence. 2003. Leaf litter decomposition of tree species in three successional phases of tropical dry secondary forest in Campeche, Mexico. Forest Ecology and Management 174(1): 401-412. 
Apéndice 1. Especies arbóreas y sus valores de importancia relativa en cada uno de los seis fragmentos estudiados. $\mathrm{G}=\mathrm{Grupo}$ florístico: $\mathrm{C}=$ conífera, $\mathrm{L}=$ latifoliada. $\mathrm{BJ}=$ Benito Juárez, $\mathrm{PC}=$ Poblado Cambil, $\mathrm{EC}=\mathrm{El}$ Carrizal, $\mathrm{LF}=\mathrm{Libertad}$ Frontera, $\mathrm{PG}=\mathrm{Plan}$ Grande y VG = Vicente Guerrero. Nomenclatura de acuerdo a The Plant List (http://www.theplantlist.org/)

Tree species and their importance value index in each of the six forest fragments studied. $\mathrm{G}=$ floristic group $\mathrm{C}=$ conifer, $\mathrm{L}=$ broadleaved. BJ: Benito Juarez, PC = Poblado Cambil, EC = El Carrizal, LF = Libertad Frontera, PG = Plan Grande y VG = Vicente Guerrero. Nomenclature according to The Plant List (http://www.theplantlist.org/).

\begin{tabular}{|c|c|c|c|c|c|c|c|c|}
\hline Familia & Especie & G & BJ & $\mathrm{PC}$ & EC & LF & PG & VG \\
\hline Actinidiaceae & Saurauia scrabida Hemsl. & $\mathrm{L}$ & 0,63 & - & - & - & - & \\
\hline Adoxaceae & Viburnum lautum C.V.Morton & $\mathrm{L}$ & 0,41 & - & - & - & - & - \\
\hline Anacardiaceae & Pistacia mexicana Kunth & $\mathrm{L}$ & - & 0,34 & 0,49 & - & - & - \\
\hline Apocynaceae & Plumeria rubra $\mathrm{L}$. & $\mathrm{L}$ & - & 0,40 & - & - & - & - \\
\hline \multirow[t]{3}{*}{ Aquifoliaceae } & Ilex costaricensis Donn.Sm. & $\mathrm{L}$ & 0,38 & - & - & - & - & - \\
\hline & Ilex discolor Hemsl. & $\mathrm{L}$ & 1,00 & - & - & - & - & - \\
\hline & Ilex macfadyenii (Walp.) Rehder & $\mathrm{L}$ & 0,17 & - & - & - & - & - \\
\hline \multirow[t]{6}{*}{ Araliaceae } & Dendropanax arboreus (L.) Decne. et Planch. & $\mathrm{L}$ & 0,96 & - & - & - & - & - \\
\hline & Oreopanax echinops (Schltdl. et Cham.) Decne. et Planch. & $\mathrm{L}$ & 1,00 & - & - & - & - & - \\
\hline & Oreopanax geminatus Marchal & $\mathrm{L}$ & - & 1,49 & - & - & - & - \\
\hline & Oreopanax liebmannii Marchal & $\mathrm{L}$ & 0,49 & - & - & - & - & - \\
\hline & Oreopanax peltatus Linden ex Regel & $\mathrm{L}$ & 0,39 & - & - & - & - & - \\
\hline & Oreopanax xalapensis (Kunth) Decne. et Planch. & $\mathrm{L}$ & 0,89 & - & - & - & - & - \\
\hline \multirow[t]{9}{*}{ Asteraceae } & Ageratina areolaris (DC.) Gage ex B.L.Turner & $\mathrm{L}$ & - & - & - & - & - & 0,39 \\
\hline & Baccharis vaccinioides Kunth & $\mathrm{L}$ & - & - & - & 1,13 & - & - \\
\hline & Critoniadelphus nubigenus (Benth.) R.M.King et H.Rob. & $\mathrm{L}$ & 0,93 & - & - & - & - & - \\
\hline & Perymenium grande Hemsl. & $\mathrm{L}$ & 2,88 & 1,40 & - & - & - & - \\
\hline & Sinclairia discolor Hook. et Arn. & $\mathrm{L}$ & 0,17 & 0,95 & - & - & - & 0,24 \\
\hline & Verbesina perymenioides Sch.Bip. ex Klatt & $\mathrm{L}$ & 1,25 & 0,64 & 0,48 & - & - & - \\
\hline & Vernonia canescens $(\mathrm{L}$.$) Sw.$ & $\mathrm{L}$ & - & - & - & 2,23 & - & - \\
\hline & Vernonia leiocarpa (DC.) Gleason & $\mathrm{L}$ & 1,53 & 1,93 & 1,21 & - & - & 3,98 \\
\hline & Vernonia scorpioides (Lam.) H.Rob. & $\mathrm{L}$ & 1,32 & - & 0,41 & - & - & - \\
\hline Betulaceae & Carpinus caroliniana Walter & $\mathrm{L}$ & 3,80 & - & - & - & - & 0,62 \\
\hline Bignoniaceae & Tecoma stans (L.) Juss. ex Kunth & $\mathrm{L}$ & - & 3,87 & - & - & - & - \\
\hline Boraginaceae & Cordia curassavica (Jacq.) Roem. et Schult. & $\mathrm{L}$ & - & 0,51 & - & - & - & - \\
\hline \multirow[t]{3}{*}{ Burseraceae } & Bursera bipinnata (Moc. et Sessé ex DC.) Engl. & $\mathrm{L}$ & - & 8,07 & - & - & - & - \\
\hline & Bursera excelsa (Kunth) Engl. & $\mathrm{L}$ & - & 0,78 & - & - & - & - \\
\hline & Bursera simaruba (L.) Sarg. & $\mathrm{L}$ & 3,79 & 2,31 & - & - & - & - \\
\hline Cannabaceae & Trema micrantha $(\mathrm{L}$.$) Blume$ & $\mathrm{L}$ & 0,75 & 5,07 & - & - & - & - \\
\hline Celastraceae & Quetzalia contracta (Lundell) Lundell & $\mathrm{L}$ & 2,07 & - & 0,87 & - & - & - \\
\hline Chloranthaceae & Hedyosmum mexicanum C. Cordem. & $\mathrm{L}$ & 0,14 & - & - & - & - & - \\
\hline \multirow[t]{2}{*}{ Clethraceae } & Clethra chiapensis L.M. González & $\mathrm{L}$ & 6,46 & - & 0,30 & - & - & - \\
\hline & Clethra mexicana DC. & $\mathrm{L}$ & 1,68 & - & - & - & - & - \\
\hline Clusiaceae & Clusia salvinii Donn.Sm. & $\mathrm{L}$ & 0,34 & - & 0,46 & - & - & - \\
\hline Cornaceae & Cornus disciflora Moc. et Sessé ex DC. & $\mathrm{L}$ & 0,27 & - & - & - & - & - \\
\hline \multirow[t]{2}{*}{ Cupressaceae } & Cupressus lusitanica Mill. & $\mathrm{C}$ & - & - & - & - & - & 0,57 \\
\hline & Juniperus comitana Martínez & $\mathrm{C}$ & 2,47 & - & - & - & - & - \\
\hline
\end{tabular}


Continuación Apéndice 1.

\begin{tabular}{|c|c|c|c|c|c|c|c|c|}
\hline Ericaceae & Arbutus xalapensis Kunth & $\mathrm{L}$ & - & - & 4,39 & - & - & 2,51 \\
\hline \multirow[t]{3}{*}{ Euphorbiaceae } & Acalypha macrostachya Jacq. & $\mathrm{L}$ & 0,60 & - & - & - & - & - \\
\hline & Bernardia dodecandra (Sessé ex Cav.) Govaerts & L & 1,07 & - & - & - & - & - \\
\hline & Sapium macrocarpum Müll.Arg. & $\mathrm{L}$ & 0,18 & - & - & - & - & - \\
\hline \multirow[t]{8}{*}{ Fabaceae } & Acacia pennatula (Schltdl. et Cham.) Benth. & $\mathrm{L}$ & - & 6,22 & 0,37 & - & - & - \\
\hline & Cojoba arborea (L.) Britton et Rose & $\mathrm{L}$ & 1,90 & - & - & - & - & - \\
\hline & Diphysa americana (Mill.) M.Sousa & $\mathrm{L}$ & 1,29 & 1,30 & 1,29 & - & - & 0,39 \\
\hline & Erythrina chiapasana Krukoff & $\mathrm{L}$ & - & 0,53 & 1,56 & - & - & 0,19 \\
\hline & Inga oerstediana Benth. & $\mathrm{L}$ & 0,25 & - & - & - & - & - \\
\hline & Inga punctata Willd. & $\mathrm{L}$ & 0,77 & - & - & - & - & - \\
\hline & Leucaena diversifolia (Schltdl.) Benth. & $\mathrm{L}$ & - & - & 0,27 & - & - & - \\
\hline & Lysiloma divaricatum (Jacq.) J.F.Macbr. & $\mathrm{L}$ & - & 3,92 & 2,27 & - & - & - \\
\hline \multirow[t]{11}{*}{ Fagaceae } & Quercus acutifolia Née & $\mathrm{L}$ & - & - & - & 6,65 & 6,34 & - \\
\hline & Quercus benthamii A.DC. & $\mathrm{L}$ & 8,17 & - & - & - & - & - \\
\hline & Quercus candicans Née & $\mathrm{L}$ & - & - & - & - & - & 1,90 \\
\hline & Quercus castanea Née & $\mathrm{L}$ & 0,70 & - & 5,25 & - & & 20,89 \\
\hline & Quercus conspersa Benth. & $\mathrm{L}$ & - & - & 0,24 & - & - & 0,77 \\
\hline & Quercus crassifolia Bonpl. & $\mathrm{L}$ & - & - & 15,87 & - & - & - \\
\hline & Quercus ocoteifolia Liebm. & $\mathrm{L}$ & - & - & 0,75 & - & - & 3,89 \\
\hline & Quercus peduncularis Née & $\mathrm{L}$ & - & 46,25 & 6,47 & 56,55 & 5,11 & 1,39 \\
\hline & Quercus polymorpha Schltdl. et Cham. & $\mathrm{L}$ & - & - & - & - & 22,07 & - \\
\hline & Quercus segoviensis Liebm. & $\mathrm{L}$ & - & 3,45 & 17,36 & - & & 27,90 \\
\hline & Quercus vicentensis Trel. & $\mathrm{L}$ & - & - & 0,12 & - & - & 2,36 \\
\hline \multirow[t]{3}{*}{ Lauraceae } & Cinnamomum zapatae Lorea-Hern. & $\mathrm{L}$ & 0,38 & - & - & - & - & - \\
\hline & Licaria capitata (Cham. et Schltdl.) Kosterm. & $\mathrm{L}$ & 0,64 & - & - & - & - & - \\
\hline & Persea americana Mill. & $\mathrm{L}$ & 2,44 & - & - & - & - & - \\
\hline Malpighiaceae & Bunchosia biocellata Schltdl. & $\mathrm{L}$ & - & - & 1,54 & - & - & - \\
\hline \multirow[t]{5}{*}{ Malvaceae } & Ceiba acuminata (S.Watson) Rose & $\mathrm{L}$ & - & 2,46 & - & - & - & - \\
\hline & Heliocarpus appendiculatus Turcz. & $\mathrm{L}$ & 0,41 & - & - & - & - & - \\
\hline & Heliocarpus donnellsmithii Rose & $\mathrm{L}$ & - & 3,35 & 0,48 & - & - & 1,55 \\
\hline & Heliocarpus terebinthinaceus (DC.) Hochr. & $\mathrm{L}$ & - & 0,44 & - & - & - & - \\
\hline & Hibiscus sp. & $\mathrm{L}$ & 0,25 & - & - & - & - & - \\
\hline \multirow[t]{2}{*}{ Melastomataceae } & Miconia desmantha Benth. & $\mathrm{L}$ & 0,24 & - & - & - & - & - \\
\hline & Miconia glaberrima (Schltdl.) Naudin & $\mathrm{L}$ & 0,09 & - & - & - & - & - \\
\hline \multirow[t]{2}{*}{ Meliaceae } & Cedrela salvadorensis Standl. & $\mathrm{L}$ & 3,67 & 1,70 & 0,51 & 0,64 & - & - \\
\hline & Trichilia havanensis Jacq. & $\mathrm{L}$ & 0,17 & - & - & - & - & - \\
\hline Monimiaceae & Mollinedia viridiflora Tul. & $\mathrm{L}$ & 0,50 & - & - & - & - & - \\
\hline Moraceae & Ficus pertusa L.f. & $\mathrm{L}$ & - & 0,96 & - & - & - & - \\
\hline Myricaceae & Morella cerifera (L.) Small & $\mathrm{L}$ & 0,81 & - & - & - & - & 1,27 \\
\hline Myrtaceae & Eugenia capuliodes Lundell & $\mathrm{L}$ & 3,40 & - & 0,53 & - & - & - \\
\hline \multirow[t]{2}{*}{ Onagraceae } & Fuchsia splendens Zucc. & $\mathrm{L}$ & 1,05 & - & - & - & - & - \\
\hline & Hauya elegans DC. & $\mathrm{L}$ & - & - & 1,37 & - & - & - \\
\hline
\end{tabular}


Continuación Apéndice 1.

\begin{tabular}{|c|c|c|c|c|c|c|c|c|}
\hline Papaveraceae & Bocconia arborea S.Watson & $\mathrm{L}$ & - & - & 0,39 & - & - & - \\
\hline Pentaphylacaceae & Symplococarpon purpusii (Brandegee) Kobuski & $\mathrm{L}$ & 0,09 & - & - & - & - & - \\
\hline Phyllonomaceae & Phyllonoma laticuspis (Turcz.) Engl. & $\mathrm{L}$ & 0,51 & - & - & - & - & - \\
\hline Picramniaceae & Alvaradoa amorphoides Liebm. & $\mathrm{L}$ & - & 1,67 & - & - & - & - \\
\hline \multirow[t]{6}{*}{ Pinaceae } & Pinus devoniana Lindl. & $\mathrm{C}$ & - & - & 2,59 & 24,29 & 38,82 & 3,53 \\
\hline & Pinus maximinoi H.E.Moore & $\mathrm{C}$ & 1,55 & - & 7,93 & - & - & 17,66 \\
\hline & Pinus montezumae Lamb. & $\mathrm{C}$ & - & - & - & - & 23,98 & - \\
\hline & Pinus oocarpa Schiede & $\mathrm{C}$ & 4,20 & - & - & 8,60 & - & - \\
\hline & Pinus pseudostrobus Lindl. & $\mathrm{C}$ & - & - & 1,22 & - & - & 4,74 \\
\hline & Pinus tecunumanii F.Schwerdtf. ex Eguiluz et J.P.Perry & $\mathrm{C}$ & 1,04 & - & 14,11 & - & 3,67 & 3,26 \\
\hline Piperaceae & Piper psilorachis C.DC. & $\mathrm{L}$ & 0,46 & - & - & - & - & - \\
\hline Podocarpaceae & Podocarpus matudae Lundell & $\mathrm{C}$ & 0,25 & - & - & - & - & - \\
\hline Polygonaceae & Coccoloba tuerckheimii Donn.Sm. & $\mathrm{L}$ & 3,03 & - & - & - & - & - \\
\hline \multirow[t]{2}{*}{ Primulaceae } & Ardisia nigrescens Oerst. & $\mathrm{L}$ & 0,19 & - & - & - & - & - \\
\hline & Myrsine coriacea (Sw.) R.Br. ex Roem. et Schult. & $\mathrm{L}$ & 2,30 & - & - & - & - & - \\
\hline Rhamnaceae & Frangula discolor (Donn.Sm.) Grubov & $\mathrm{L}$ & - & - & 0,22 & - & - & - \\
\hline \multirow[t]{4}{*}{ Rosaceae } & Prunus brachybotrya Zucc. & $\mathrm{L}$ & 2,26 & - & - & - & - & - \\
\hline & Prunus lundelliana Standl. & $\mathrm{L}$ & 0,34 & - & - & - & - & - \\
\hline & Prunus rhamnoides Koehne & $\mathrm{L}$ & 0,31 & - & - & - & - & - \\
\hline & Prunus serotina Ehrh. & $\mathrm{L}$ & - & - & 0,22 & - & - & - \\
\hline \multirow[t]{4}{*}{ Rubiaceae } & Arachnothryx buddleioides (Benth.) Planch. & $\mathrm{L}$ & 2,09 & - & - & - & - & - \\
\hline & Chiococca alba (L.) Hitchc. & $\mathrm{L}$ & 4,80 & - & 0,19 & - & - & - \\
\hline & Glossostipula concinna (Standl.) Lorence & $\mathrm{L}$ & 0,57 & - & 1,28 & - & - & - \\
\hline & Rogiera aprica (Lundell) Borhidi & $\mathrm{L}$ & 1,31 & - & 1,27 & - & - & - \\
\hline \multirow[t]{2}{*}{ Rutaceae } & Zanthoxylum riedelianum Engl. & $\mathrm{L}$ & 0,25 & - & - & - & - & - \\
\hline & Zanthoxylum sp. & $\mathrm{L}$ & 0,25 & - & - & - & - & - \\
\hline Solanaceae & Solanum nigricans $\mathrm{M}$. Martens et Galeotti & $\mathrm{L}$ & 1,46 & - & 1,28 & - & - & - \\
\hline Styracaceae & Styrax ramirezii (Greenm.) Gonsoulin & $\mathrm{L}$ & 0,71 & - & - & - & - & - \\
\hline \multirow[t]{2}{*}{ Urticaceae } & Boehmeria ulmifolia Wedd. & $\mathrm{L}$ & 2,72 & - & - & - & - & - \\
\hline & Urera caracasana (Jacq.) Gaudich. ex Griseb. & $\mathrm{L}$ & 2,38 & - & - & - & - & - \\
\hline \multirow[t]{3}{*}{ Verbenaceae } & Citharexylum donnell-smithii Greenm. & $\mathrm{L}$ & 0,26 & - & - & - & - & - \\
\hline & Citharexylum mocinni D.Don & $\mathrm{L}$ & 1,54 & - & 1,85 & - & - & - \\
\hline & Lippia umbellata Cav. & $\mathrm{L}$ & - & - & 2,60 & - & - & - \\
\hline
\end{tabular}

